\title{
Contrast-enhanced spectral mammography: successful initial clinical institute experience
}

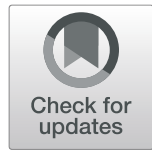

Nivine Abdel Moneim Chalabi, Amal Amin AbuEIMaati and Momena Essam Ibrahim Elsadawy*

\begin{abstract}
Background: Contrast-enhanced spectral mammography (CESM) is a relatively newly developed advanced application with modification of digital mammography by the use of a contrast agent, but still has little known efficacy among Egyptian patients. Our aim in this study is to share our initial experience in evaluating symptomatic patients with different ACR breast parenchyma especially in dense breast parenchyma as it is always challenging in diagnosis.

Results: CESM in this study gave a sensitivity of $92 \%$ and specificity of $85 \%$ in characterization of benign and malignant lesions. For postoperative cases, sensitivity was $85 \%$ and specificity was $60 \%$. For chemotherapy cases, sensitivity was $85 \%$ and specificity was $76 \%$. Contrast uptake was noted in $68 \%$ of masses. Cavitary benign lesions were noted in $22.1 \%$ of cases. Multifocal and multicentric carcinomas were detected in $39.7 \%$ of pathologically proved malignant masses. Statistical analysis revealed sensitivity, specificity, and accuracy of $82.9 \%, 76.5 \%$, and $81.0 \%$ for conventional mammograms as compared to $92.7 \%, 82.4 \%$, and $89.7 \%$ for CESM respectively.

Conclusion: CESM is a promising technique that can enhance the specificity of conventional mammograms. It is an easy, simple, and rapid contrast-based procedure, especially for characterization of lesions in dense breast parenchyma. It performs proper diagnosis for high-risk patients and follow-up response to different lines of management.
\end{abstract}

Keywords: Dense breast, Breast cancer, Contrast-enhanced spectral mammography (CESM)

\section{Background}

Full-field digital mammography (FFDM) is a rapid procedure with high-quality images and higher contrast resolution with improved dynamic range, and rapid processing of data and images as compared to screen-film mammography $[1,2]$. Its diagnostic accuracy has been shown to be at least equivalent to film-screen mammography $[1,3]$. However, some limitations still exist, mainly in dense breast tissue, fibrocystic disease, and during follow-up after breast-conserving/adjuvant therapy. A new procedure has been developed using contrast media for the detection of angiogenesis by tracing contrast agent uptake and washout in suspicious lesions. Contrast-enhanced spectral mammography (CESM) is one of them $[4,5]$. CESM provides data about the degree of vascularization of the lesion in addition to morphological information. According to recent studies, CESM is considered superior

\footnotetext{
* Correspondence: momena.essam@yahoo.com
}

Ain Shams University, Cairo, Egypt

\section{Springer Open}

(c) The Author(s). 2021 Open Access This article is licensed under a Creative Commons Attribution 4.0 International License, which permits use, sharing, adaptation, distribution and reproduction in any medium or format, as long as you give appropriate credit to the original author(s) and the source, provide a link to the Creative Commons licence, and indicate if changes were made. The images or other third party material in this article are included in the article's Creative Commons licence, unless indicated otherwise in a credit line to the material. If material is not included in the article's Creative Commons licence and your intended use is not permitted by statutory regulation or exceeds the permitted use, you will need to obtain permission directly from the copyright holder. To view a copy of this licence, visit http://creativecommons.org/licenses/by/4.0/.

to conventional mammography in breast cancer detection with less false negatives especially in dense breasts [6]. A clinical study by Dromain et al. [7] showed that digital mammography plus CESM had higher sensitivity in the detection of suspicious lesions in dense breasts.

Contrast-enhanced breast MRI is currently considered the most sensitive imaging technique for the detection of breast carcinoma and hence, has many indications in diagnosis, treatment and follow-up of breast cancer cases [8]. However, MRI has limited specificity, widely available, expensive, and not suitable for claustrophobic patients.

In August 2017, our Pristina Senograhe FFDM and tomosynthesis machine was installed in the outpatient clinic in our institutional hospitals.

In April 2018, we started using CESM.

From August 2018 to date, we did 123 cases with over 80 cases of biopsy-proven malignancy. In most of the 
cases, US-guided biopsy was taken on the same day and place where the study took place.

The aim of this work was to share our experience during the first year of using the CESM technique and discuss and illustrate its potential clinical applications.

\section{Methods}

The machine was first installed in August 2017 at our breast radiology outpatient clinic, which serves about 1000 patients per year, with mostly diagnostic imaging services. The machine installed was a GE Senographe Pristina mammography system, the first to be installed in Egypt and the whole of Africa. Our practice during the first year of the use of dual-energy contrastenhanced spectral mammography spanning from April 2018 to March 2019 was assessed.

\section{Patients}

This study was approved by the local institutional review board. Objectives and methodology were clearly explained to the patients, written informed consent was provided to all participating patients.

Our challenge, like most practices, is providing valuebased care. We began using CESM in April 2018. Since then, we have used CESM in a variety of clinical applications that would have been evaluated with MRI. From April 2018 to March 2019, we enrolled 123 patients aged 27-72 years (mean 44.95 years).

The study population included women referred from the clinic for screening and diagnostic mammogram; examinations were performed for staging of proven breast cancer in 23 patients (18.7\%), clarify the nature of indeterminate lesions in 63 patients $(55.7 \%)$ that included evaluation of focal asymmetry in 24 patients (19.5\%), parenchymal distortion in 13 patients $(10.6 \%)$, and mass in dense breasts in 9 patients (7.3\%), and 17 patients (13.8\%) had a suspicious lesion on mammography with no ultrasound correlate. The examination was also done for postoperative follow-up in 12 patients (9.8\%). Patients were sent from the oncology department for follow-up of neoadjuvant chemotherapy (NAC) and 25 (20.3\%) patients were included in our study.

Patients underwent diagnostic conventional mammograms, ultrasound, and then followed by CESM. A detailed history of the patient with a review of her previous studies is done. A review of the serum creatinine as well as the history of allergy or previous reaction is noted.

Exclusion criteria included pregnancy, breast implants, renal insufficiency, and history of allergic reaction to iodinated contrast agents.

\section{Digital mammography}

All patients performed digital mammography (including craniocaudal (CC) and mediolateral oblique (MLO) views) and were evaluated retrospectively to CESM interpretation.

\section{Contrast-enhanced digital mammography examination}

The procedure was done in two steps: contrast administration and imaging. A catheter was placed into the antecubital vein of the contralateral arm to the affected breast. A dose of $300 \mathrm{mg} \mathrm{I} / \mathrm{ml}$ of non-ionic iodinated contrast agent, iopromide (Ultravist), was administrated. It was delivered intravenously as a single shot to the patient at a dose of $1.5-\mathrm{mmol} / \mathrm{Kg}$ body weight. CESM imaging proceeds at $2 \mathrm{~min}$ after the initiation of the contrast agent administration, the "normal" breast was compressed first in a CC and MLO views and a dualenergy of low- and high-energy images was acquired. The procedure was followed by CC and MLO views for the concerned breast to increase the likelihood of contrast uptake. After the CESM examination, patients were observed for $30 \mathrm{~min}$ for the possibility of any allergic reaction to iodinated contrast agents. The first low-energy $(26-30 \mathrm{kVp})$ image is a standard 2D mammogram and the second high energy $(45-49 \mathrm{kVp})$ image is a high- $\mathrm{kV}$ mammogram. Then, the two images are combined to enhance areas of contrast uptake. The total duration of the examination was $7 \mathrm{~min}$.

Senographe Pristina is FDA-approved mammography and delivers CESM at the same low dose as 2D FFDM.

\section{Image analysis}

Two experienced, independent radiologists with 10 years' interpretation experience in breast imaging have evaluated all lesions. Digital mammography images were evaluated using BI-RADS (ACR) lexicon assessment.

The radiologists reviewed the processed images in the workstation. A dual-energy CESM examination provides two images of each view, one at low energy which is similar to conventional mammography with a high contrast-to-noise ratio of micro calcifications and the other one acquired at high energy giving a high contrast-to-noise ratio of iodine. Analysis of low-energy images was completed regarding the following: breast density, location, and type of each finding (asymmetry, mass, density, calcification, asymmetry, architectural distortions, intramammary lymph nodes, and scars) according to the 2013 BI-RADS (ACR) lexicon [9].

After that, the subtracted images are reviewed using the reading criteria based on lesion morphology description as well as the degree of contrast enhancement intensity in comparison to background enhancement. Lesions on CESM were described using the same MRI description according to the 2013 MRI BI-RADS lexicon as there is no CESM specific lexicon [10]. The degree of background parenchymal enhancement was reported, and lesions were classified into non-enhancing, mild, moderate, and 
markedly enhanced. Non-enhancing and mildly enhancing lesions usually reflect benign morphology and subjected to follow-up. Moderately and markedly enhancing lesions showing malignant morphology and were subjected to immediate core biopsy.

Patients with suspicious findings underwent ultrasoundguided or stereotactic-guided core biopsy. US-guided biopsy was obtained on the same day and place where the study took place. Written patient consent was obtained for all biopsy procedures.

Cystic lesions were subjected to ultrasound-guided fine-needle aspiration and cytological assessment.

Histopathological results were collected from surgery for patients who were under follow-up of neoadjuvant chemotherapy and also for patients who underwent examination for the staging of proven breast cancer.

Patients without suspicious findings were subjected to follow-up every 6 months to assure the absence of cancer.

\section{Statistical analysis}

MedCalc version 18.2.1 (MedCalc Software, Ostend, Belgium) and XLSTAT version 2014.5.03 (Addinsoft, NY, USA) systems were used for data analysis.

The histopathological results (or follow-up) were the gold standard tests.

The following diagnostic indices were calculated: specificity, sensitivity, positive and negative predictive values, and positive and negative likelihood ratios, as well as correct classification and misclassification rates.

Inter-method agreement for binary outcomes was examined by calculation of Cohen's kappa ( $\kappa$ ), Scott's biasadjusted kappa (BAK, $\pi$ ), and Bennett's prevalenceadjusted bias-adjusted kappa (PABAK). Agreement for ordinal variables was examined using the weighted kappa coefficient. A $p$-value less than 0.05 was considered statistically significant.

\section{Results}

We retrospectively analyzed CESM of 123 patients with 133 lesions (3 multifocal and 7 multicentric IDC). We considered the histopathological results of the surgical piece as the gold standard for the 49 malignant lesions obtained from the patients who underwent surgery. True cut biopsy was done for 56 cases and was correlated with the histopathological results as a gold standard. Cytology results of 5 benign cystic lesions was employed as the gold standard. Follow-up was performed on the remaining 13 benign lesions every 6 months. According to histopathology and outcome of follow-up, 86 patients proved to have malignant pathology and 37 had benign pathology.

Breast parenchymal density was evaluated on the low energy mammographic images: 49/123 (39.8\%) cases were classified as ACR "a" or "b" and 74/123 (60.1\%) were classified as ACR "c" or "d."

Further assessment was performed for staging of proved malignancy in $18.7 \%$ of cases $(n=23)$ and to clarify the nature of indeterminate lesions including suspicious postoperative cases in another $61.7 \%(n=76)$. Follow-up cases post neoadjuvant chemotherapy were $20.3 \%(n=25)$.

Benign lesions (in order of frequency) included: 8 fibroadenomas (10.6 \%) (Fig. 1); 5 benign postsurgical changes (13.5\%); 5 cysts (13.5\%); 4 cases granulomatous mastitis (10.8\%); 3 sclerosing adenosis (8.1\%); 3 fibrocystic lesions (8.1\%); 3 fat necrosis (8.1\%); 3 atypical ductal hyperplasia (8.1\%); 2 intramammary lymph nodes (5.4\%); 2 papilloma (5.4\%); 1 hamartoma (2.7\%); and 1 lipoma (2.7\%).

Malignant lesions included: 25 invasive ductal carcinoma (33.25\%); 13 invasive lobular carcinoma (15.1\%) (Fig. 2); 11 cases with ductal carcinoma in situ (DCIS) (12.7\%); 11 IDC with in situ component (12.7\%); and 2 cases of mucinous carcinoma (2.3\%). 25 cases were NAC under follow-up (33.25\%) and 4 cases of recurrent IDC were diagnosed among cases under postoperative followup, 2 cases were false positive cases of sclerosing adenosis.

\section{Statistical methods}

Data were analyzed using MedCalc@ version 18.2.1 (MedCalc@ Software bvba, Ostend, Belgium) and

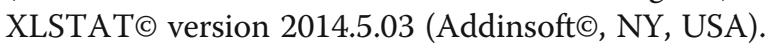

Categorical data were presented as number and percentage and inter-group differences were compared using the Pearson chi-squared test (for nominal data) or the chi-squared test for trend (for ordinal data).

The diagnostic value of radiological tools for discrimination between malignant and benign lesions was examined versus the result of histopathology (or follow-up) as the gold standard test. The following diagnostic indices were calculated: sensitivity, specificity, positive and negative predictive values, positive and negative likelihood ratios, and correct classification and misclassification rates.

Inter-method agreement for binary outcomes was examined by calculation of Cohen's kappa $(\kappa)$, Scott's biasadjusted kappa (BAK, $\pi$ ), and Bennett's prevalenceadjusted bias-adjusted kappa (PABAK). Agreement for ordinal variables was examined using the weighted kappa coefficient. The agreement coefficients are interpreted as follows in Table 1.

Variable parameters of whole study population characteristics are included in Table 2.

The radiological characteristics of benign and malignant lesions are assessed and included in Table 3.

Sonomammography in our study showed sensitivity of $93 \%$ and $67 \%$ specificity as in Table 4 . 

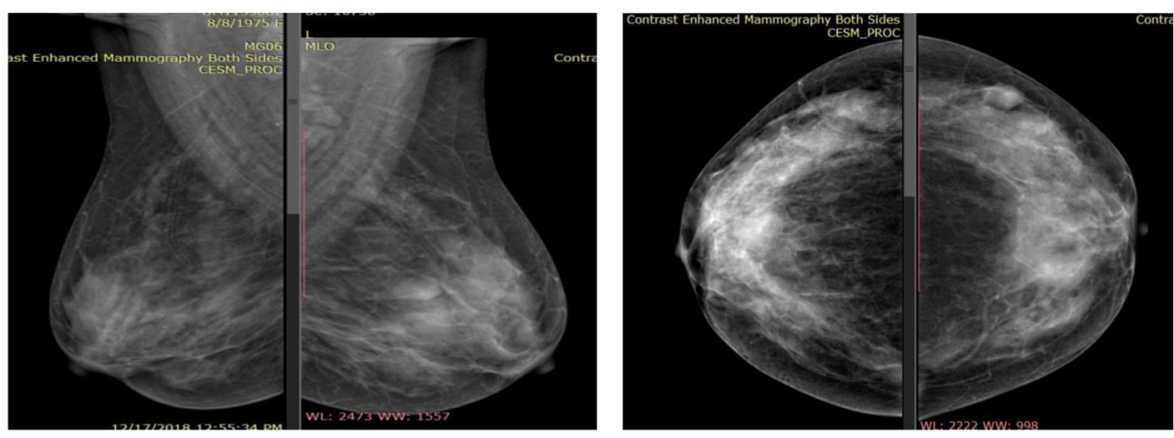

(A)
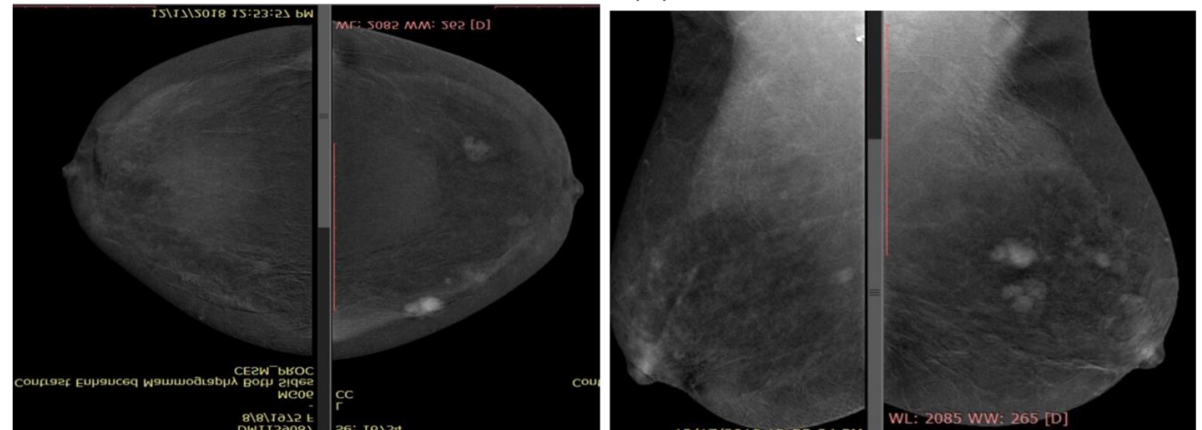

(B)

Fig. 1 44-year-old female with left palpable mass. A CC and MLO low-energy CESM images showed heterogenously dense breasts with asymmetric density seen at lateral left breast. B CC and MLO recombined images showed multiple bilateral enhancing lesions that were revealed to be fibrosclerotic tissue core with focal fibroadenomatous hyperplasia

Sonomammography showed $98 \%$ sensitivity and $71 \%$ specificity in ACR A/B breast parenchymal density as shown in Table 5.

Sonomammography assessed lesions in ACR grade C/ $\mathrm{D}$ and showed $92 \%$ sensitivity and $72 \%$ specificity as shown in Table 6.

CESM gave $100 \%$ sensitivity of diagnostic accuracy and $89 \%$ specificity as in Table 7 .

CESM showed 100 sensitivity and $92 \%$ specificity when tested in patients with ACR A/B as shown in Table 8.

CESM showed $100 \%$ sensitivity and $91 \%$ specificity when tested in patients with ACR grade C/D as in Table 9.

\section{CESM in staging of proved malignancy}

An $18.7 \%$ of the cases included in our study $(n=23)$ were pathologically proven to be malignant (BI-RADS 6) and were referred for staging. The accuracy of sonomammography and CESM regarding lesions multiplicity was calculated (Table 1, Fig. 3) The gold standard reference for malignant lesions multiplicity was the histopathological analysis of the surgical specimen.

CESM showed high sensitivity as regards the identification of multiplicity reaching up to $99.7 \%$ (with little limitation due to breast density) but with specificity of 97.3\% as some benign lesions could display a significant enhancement such as in inflammatory lesions, some benign tumors like fibroadenoma and, intra ductal papillomas. CESM showed a good positive predictive value of $96.4 \%$ and negative predictive value of $98 \%$ (Table 10 , $11,12,13,14,15,16,17$ and 18).

\section{CESM for evaluation of indeterminate lesions}

CESM findings of 76 suspicious or indeterminate findings in 123 patients done at our "Radiology outpatient breast clinic" were retrospectively analyzed. 17 patients had suspicious lesions on mammography with no correlated ultrasound findings, 9 patients had lesions in dense breast, 13 patients with parenchymal distortion, 24 patients had focal asymmetry, and 12 patients were subjected to postoperative follow-up.

According to histopathology results and outcomes of follow-up studies, 31/76 (41.3\%) patients turned out to be benign or probably benign lesions (BI-RADS 2 and 3), while $45 / 76(59.2 \%)$ patients proved to be malignant lesions (BI-RADS 4 and 5).

Asymmetries were then classified into single view (6\%), focal $(82 \%)$, global (4\%), and developing (8\%). The presence of global, focal, or developing asymmetry are strongly correlated with an underlying malignant pathology ( $p$ value 0.001 ) (Table 2). Associated mammography findings 


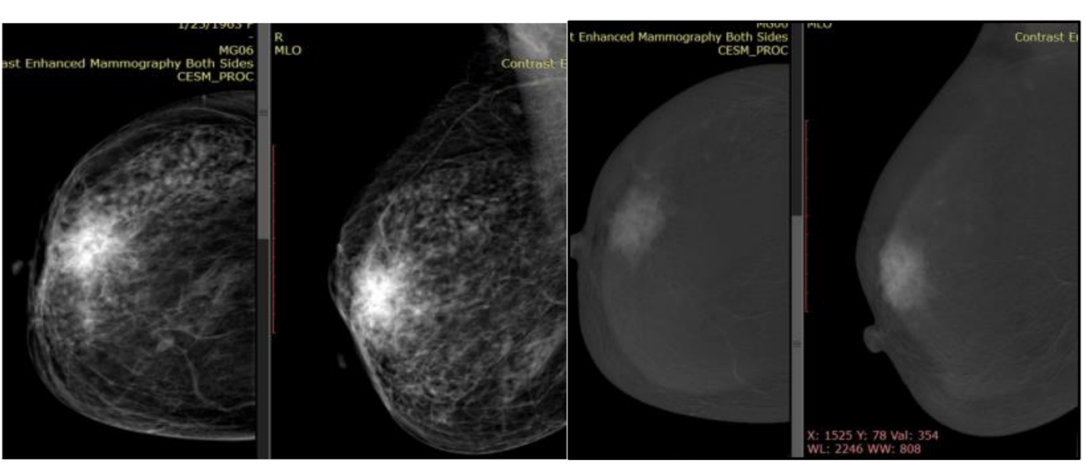

(A)

(B)

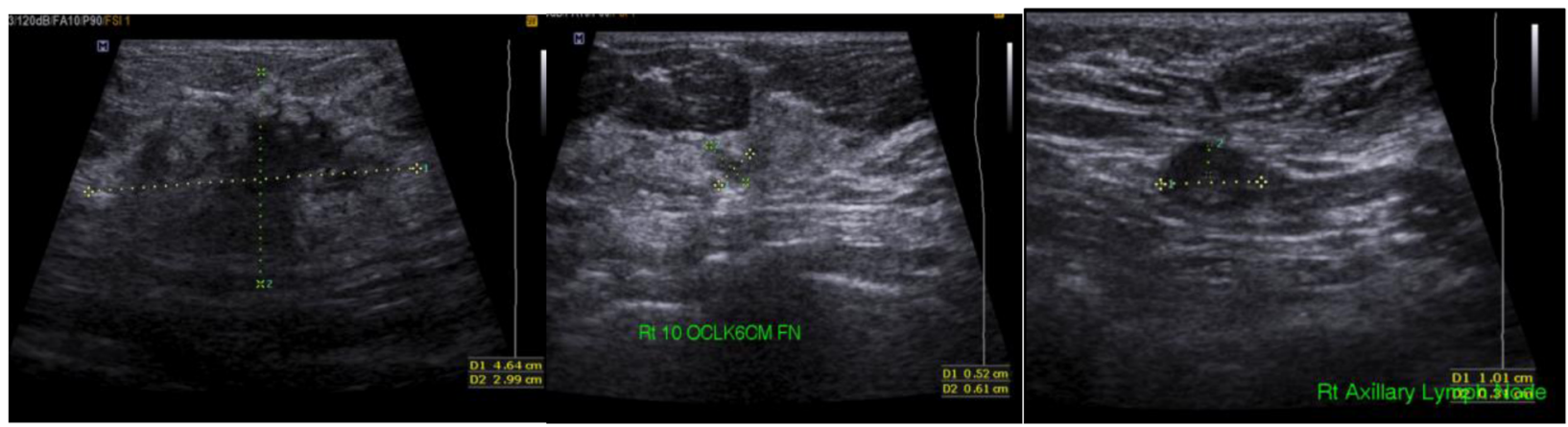

(C)

(D)

(E)

Fig. 2 56-year-old female patient with right palpable lump. A Low-energy CEDM, MLO, and CC views of the right side showed retroareolar irregular spiculated mass lesion. B Recombined CEDM image showed avid enhancement of that lesion, with other enhancing nodules seen in the upper outer quadrant. C Targeted ultrasound examination revealed irregularly shaped spiculated hypoechoic mass lesion and another smaller one at the upper outer quadrant corresponding to the enhancing nodule detected. D Suspicious thickened cortex axillary lymph node is noted as well. E Histopathology revealed invasive lobular carcinoma (ILC)

(e.g., skin thickening, edema, calcification, and parenchymal distortion) were seen in 56 cases (78\%) cases. On evaluating the low-energy mammographic images, a considerable number of false negative (36\%) and false positive (48\%) and false negative (36\%) asymmetries were encountered.

\section{CESM in following up cases under neoadjuvant chemotherapy}

As regards patients with locally advanced breast cancer under neoadjuvant chemotherapy for follow-up $(n=25)$

Table 1 Agreement for ordinal variables was examined using the weighted kappa coefficient. The agreement coefficients are interpreted as follows

\begin{tabular}{ll}
\hline Coefficient of agreement & Strength of agreement \\
\hline $0.0-0.20$ & Slight agreement \\
$0.21-0.40$ & Fair agreement \\
$0.41-0.60$ & Moderate agreement \\
$0.61-0.80$ & Substantial agreement \\
$0.81-1.0$ & Nearly perfect agreement \\
\hline
\end{tabular}

$p$-values $<0.05$ were considered statistically significant
(20.3\%), initial bilateral mammography and complementary US were done (or reviewed if available) in order to determine the tumor location and its baseline size. Insertion of pretherapy clips was done to localize the tumor for further follow-up and assessment of therapy response (Fig. 4).

Interpretation was done using response evaluation criteria in solid tumors (RECIST) guidelines for evaluation of target lesions [11]: that was agreed upon with the oncology department during our weekly meeting.

Complete response (CR): complete resolution of all main lesions with reduction of the short axis of pathological lymph nodes to $<10 \mathrm{~mm}$.

Partial response (PR): at least $30 \%$ reduction in the diameters of the main lesion.

Progressive disease (PD): At least 20\% increase in the diameters of main lesions when compared to the smallest sum diameters. De novo lesions are also considered progression.

Stable disease (SD): the lesion remains stable in diameter as compared to the smallest sum diameters of the original lesion.

The patterns of contrast enhancement of the residual tumors had various patterns and were interpreted as 
Table $\mathbf{2}$ Characteristics of the whole study population

\begin{tabular}{|c|c|c|c|}
\hline \multicolumn{2}{|l|}{ Variable } & \multirow{2}{*}{$\frac{N}{44}$} & \multirow{2}{*}{$\frac{\%}{35.8 \%}$} \\
\hline Age category & $\leq 40$ years & & \\
\hline & $>40$ years & 79 & $64.2 \%$ \\
\hline \multirow[t]{4}{*}{ ACR grade } & Grade A & 4 & $3.3 \%$ \\
\hline & Grade B & 45 & $36.6 \%$ \\
\hline & Grade C & 68 & $55.3 \%$ \\
\hline & Grade D & 6 & $4.9 \%$ \\
\hline \multirow{5}{*}{$\begin{array}{l}\text { Number of lesions by } \\
\text { SMG }\end{array}$} & Nil & 6 & $4.9 \%$ \\
\hline & Single lesion & 96 & $78.0 \%$ \\
\hline & 2 lesions & 12 & $9.8 \%$ \\
\hline & 3 lesions & 5 & $4.1 \%$ \\
\hline & 4 lesions & 4 & $3.3 \%$ \\
\hline \multirow[t]{6}{*}{ BIRADS class by SMG } & BIRADS1 & 7 & $5.7 \%$ \\
\hline & BIRADS 2 & 0 & $0.0 \%$ \\
\hline & BIRADS 3 & 23 & $18.7 \%$ \\
\hline & BIRADS 4 & 40 & $32.5 \%$ \\
\hline & BIRADS 5 & 4 & $3.3 \%$ \\
\hline & BIRADS 6 & 49 & $39.8 \%$ \\
\hline \multirow[t]{8}{*}{ Indication for CESM } & $\begin{array}{l}\text { Follow-up of neo-adjuvant } \\
\text { chemotherapy }\end{array}$ & 25 & $20.3 \%$ \\
\hline & Mass in dense breast & 8 & $6.5 \%$ \\
\hline & Staging of breast cancer & 23 & $18.7 \%$ \\
\hline & $\begin{array}{l}\text { Suspicious lesion on } \\
\text { mammography with no } \\
\text { US correlate }\end{array}$ & 17 & $13.8 \%$ \\
\hline & Focal asymmetry & 24 & $19.5 \%$ \\
\hline & Postoperative follow-up & 12 & $9.8 \%$ \\
\hline & Parenchymal distortion & 13 & $10.6 \%$ \\
\hline & Suspicious palpable lesion & 1 & $0.8 \%$ \\
\hline \multirow{6}{*}{$\begin{array}{l}\text { Number of lesions by } \\
\text { CESM }\end{array}$} & Nil & 22 & $17.9 \%$ \\
\hline & Single lesion & 61 & $49.6 \%$ \\
\hline & 2 lesions & 12 & $9.8 \%$ \\
\hline & 3 lesions & 22 & $17.9 \%$ \\
\hline & 4 lesions & 5 & $4.1 \%$ \\
\hline & 5 lesions & 1 & $0.8 \%$ \\
\hline \multirow[t]{5}{*}{ Grade of enhancement } & No enhancement & 24 & $19.5 \%$ \\
\hline & Minimal & 12 & $9.8 \%$ \\
\hline & Mild & 11 & $8.9 \%$ \\
\hline & Moderate & 14 & $11.4 \%$ \\
\hline & Marked & 62 & $50.4 \%$ \\
\hline \multirow[t]{5}{*}{ BIRADS class by CESM } & BIRADS 2 & 29 & $23.6 \%$ \\
\hline & BIRADS 3 & 2 & $1.6 \%$ \\
\hline & BIRADS 4 & 37 & $30.1 \%$ \\
\hline & BIRADS 5 & 8 & $6.5 \%$ \\
\hline & BIRADS 6 & 47 & $38.2 \%$ \\
\hline
\end{tabular}

Table 2 Characteristics of the whole study population (Continued)

\begin{tabular}{|c|c|c|c|}
\hline Variable & & $N$ & $\%$ \\
\hline \multirow{23}{*}{$\begin{array}{l}\text { Pathological type and } \\
\text { course }\end{array}$} & Adenosis & 3 & $2.4 \%$ \\
\hline & Atypical ductal hyperplasia & 3 & $2.4 \%$ \\
\hline & Benign postoperative changes & 5 & $4.1 \%$ \\
\hline & Complete response & 8 & $6.5 \%$ \\
\hline & Cyst & 4 & $3.3 \%$ \\
\hline & DCIS & 11 & $8.9 \%$ \\
\hline & Fat necrosis & 3 & $2.4 \%$ \\
\hline & Fibroadenoma & 5 & $4.1 \%$ \\
\hline & Fibrocystic changes & 3 & $2.4 \%$ \\
\hline & Granulomatous mastitis & 4 & $3.3 \%$ \\
\hline & Hamartoma & 1 & $0.8 \%$ \\
\hline & IDC & 18 & $14.6 \%$ \\
\hline & $\begin{array}{l}\text { IDC within in-situ } \\
\text { component }\end{array}$ & 11 & $8.9 \%$ \\
\hline & ILC & 13 & $10.6 \%$ \\
\hline & IMLN & 2 & $1.6 \%$ \\
\hline & Infected cyst & 1 & $0.8 \%$ \\
\hline & Lipoma & 1 & $0.8 \%$ \\
\hline & Minimal residual disease & 5 & $4.1 \%$ \\
\hline & Mucinous carcinoma & 2 & $1.6 \%$ \\
\hline & Papilloma & 2 & $1.6 \%$ \\
\hline & Partial response & 2 & $1.6 \%$ \\
\hline & Recurrent IDC & 4 & $3.3 \%$ \\
\hline & Stationary disease & 12 & $9.8 \%$ \\
\hline \multirow[t]{5}{*}{ Reference index } & Follow-up & 13 & $10.6 \%$ \\
\hline & Needle aspiration & 5 & $4.1 \%$ \\
\hline & Tru-Cut biopsy & 55 & $44.7 \%$ \\
\hline & Surgical biopsy & 49 & $39.8 \%$ \\
\hline & Stereotactic biopsy & 1 & $0.8 \%$ \\
\hline \multirow[t]{2}{*}{ Final diagnosis } & Benign & 37 & $30.1 \%$ \\
\hline & Malignant & 86 & $69.9 \%$ \\
\hline
\end{tabular}

follows: No contrast uptake indicates complete therapeutic response (CR); intense contrast enhancement denotes chemo-resistance: stable disease (SD) or progressive disease (PD); faint uptake and homogenous background uptake indicate minimal residual disease and considered partial response (PR).

Histopathological diagnosis is used as the gold standard.

We had only two cases (about $2.5 \%$ of cases) showing partial response with intense CESM enhancement. Complete pathological response was achieved in $37 \%$ of cases (30 patients). CESM showed complete response in 29 out of 30 cases by showing no enhancement pattern. 
Table 3 Radiological characteristics of benign or malignant lesions

\begin{tabular}{|c|c|c|c|c|c|c|c|}
\hline \multirow[t]{3}{*}{ Variable } & & \multicolumn{4}{|c|}{ Final diagnosis } & \multirow[t]{3}{*}{$x^{2}(\mathrm{df}, 1)$} & \multirow[t]{3}{*}{$p$-value } \\
\hline & & \multicolumn{2}{|c|}{ Benign $(n=37)$} & \multicolumn{2}{|c|}{ Malignant $(n=86)$} & & \\
\hline & & $n$ & $\%$ & $n$ & $\%$ & & \\
\hline \multirow[t]{5}{*}{ Number of lesions by SMG } & $\mathrm{Nil}$ & 6 & $16.2 \%$ & 0 & $0.0 \%$ & \multirow[t]{5}{*}{14.506} & \multirow[t]{5}{*}{$<0.001^{*}$} \\
\hline & Single lesion & 31 & $83.8 \%$ & 65 & $75.6 \%$ & & \\
\hline & 2 lesions & 0 & $0.0 \%$ & 12 & $14.0 \%$ & & \\
\hline & 3 lesions & 0 & $0.0 \%$ & 5 & $5.8 \%$ & & \\
\hline & 4 lesions & 0 & $0.0 \%$ & 4 & $4.7 \%$ & & \\
\hline \multirow[t]{6}{*}{ BIRADS class by SMG } & BIRADS 1 & 4 & $10.8 \%$ & 3 & $3.5 \%$ & \multirow[t]{6}{*}{31.321} & \multirow[t]{6}{*}{$<0.001^{*}$} \\
\hline & BIRADS 2 & 0 & $0.0 \%$ & 0 & $0.0 \%$ & & \\
\hline & BIRADS 3 & 15 & $40.5 \%$ & 8 & $9.3 \%$ & & \\
\hline & BIRADS 4 & 16 & $43.2 \%$ & 24 & $27.9 \%$ & & \\
\hline & BIRADS 5 & 1 & $2.7 \%$ & 3 & $3.5 \%$ & & \\
\hline & BIRADS 6 & 1 & $2.7 \%$ & 48 & $55.8 \%$ & & \\
\hline \multirow[t]{6}{*}{ Number of enhanced lesions by CESM } & $\mathrm{Nil}$ & 14 & $37.8 \%$ & 8 & $9.3 \%$ & \multirow[t]{6}{*}{22.991} & \multirow[t]{6}{*}{$<0.001^{*}$} \\
\hline & Single lesion & 22 & $59.5 \%$ & 39 & $45.3 \%$ & & \\
\hline & 2 lesions & 0 & $0.0 \%$ & 12 & $14.0 \%$ & & \\
\hline & 3 lesions & 1 & $2.7 \%$ & 21 & $24.4 \%$ & & \\
\hline & 4 lesions & 0 & $0.0 \%$ & 5 & $5.8 \%$ & & \\
\hline & 5 lesions & 0 & $0.0 \%$ & 1 & $1.2 \%$ & & \\
\hline \multirow[t]{5}{*}{ Grade of enhancement } & No enhancement & 16 & $43.2 \%$ & 8 & $9.3 \%$ & \multirow[t]{5}{*}{25.294} & \multirow[t]{5}{*}{$<0.001^{*}$} \\
\hline & Minimal & 0 & $0.0 \%$ & 12 & $14.0 \%$ & & \\
\hline & Mild & 10 & $27.0 \%$ & 1 & $1.2 \%$ & & \\
\hline & Moderate & 8 & $21.6 \%$ & 6 & $7.0 \%$ & & \\
\hline & Marked & 3 & $8.1 \%$ & 59 & $68.6 \%$ & & \\
\hline \multirow[t]{6}{*}{ BIRADS class by CESM } & BIRADS 1 & 0 & $0.0 \%$ & 0 & $0.0 \%$ & \multirow[t]{6}{*}{71.990} & \multirow[t]{6}{*}{$<0.001^{*}$} \\
\hline & BIRADS 2 & 28 & $75.7 \%$ & 1 & $1.2 \%$ & & \\
\hline & BIRADS 3 & 1 & $2.7 \%$ & 1 & $1.2 \%$ & & \\
\hline & BIRADS 4 & 7 & $18.9 \%$ & 30 & $34.9 \%$ & & \\
\hline & BIRADS 5 & 0 & $0.0 \%$ & 8 & $9.3 \%$ & & \\
\hline & BIRADS 6 & 1 & $2.7 \%$ & 46 & $53.5 \%$ & & \\
\hline \multirow[t]{2}{*}{ BIRADS class by SMG } & Probably benign (BIRADS 1-3) & 19 & $51.4 \%$ & 11 & $12.8 \%$ & \multirow[t]{2}{*}{20.859} & \multirow[t]{2}{*}{$<0.001^{\S}$} \\
\hline & Probably malignant BIRADS 4-6 & 18 & $48.6 \%$ & 75 & $87.2 \%$ & & \\
\hline \multirow[t]{2}{*}{ BIRADS class by CESM } & Probably benign (BIRADS 1-3) & 29 & $78.4 \%$ & 2 & $2.3 \%$ & \multirow[t]{2}{*}{79.375} & \multirow[t]{2}{*}{$<0.001^{\S}$} \\
\hline & Probably malignant BIRADS 4-6 & 8 & $21.6 \%$ & 84 & $97.7 \%$ & & \\
\hline Marked enhancement & No to moderate enhancement & 34 & $91.9 \%$ & 27 & $31.4 \%$ & 37.874 & $<0.001^{\mathrm{s}}$ \\
\hline & Marked enhancement & 3 & $8.1 \%$ & 59 & $68.6 \%$ & & \\
\hline
\end{tabular}

Data are number (n) and percentage (\%). $X^{2}$, chi-squared statistic; df, 1

*Pearson chi-squared test. ${ }^{5} \mathrm{Chi}$-squared test for trend

One case showed contrast enhancement, yet had complete response evident by pathology (false positive). The specificity of CESM in our study for prediction of complete response is $68.6 \%$ and the negative predictive value is $100 \%$ with a $96 \% \mathrm{CI}=40.2-100.00 \%$.

Minimal residual disease represented $16 \%$ of the tumor responses and in 4 out of $5 \mathrm{Bcases}$ and was presented by faint enhancement pattern that was concordant to pathological response and one case showed marked tumor size reduction with faint homogenous background enhancement on CESM that also was concordant with the pathological response.

Forty-eight percent (12 cases) represented chemoresistant tumors in the final pathologic tumor response of locally advanced breast cancer, three cases showed 
Table 4 Diagnostic accuracy of sonomammography tested in the whole study cohort versus the result of biopsy or follow-up as the gold standard for lesion classification

\begin{tabular}{llll}
\hline Lesion classification by SMG & Final diagnosis & Total \\
& Malignant & Benign & 18 \\
Probably malignant (BI-RADS 4-6) & 75 & 19 & 33 \\
Probably benign (BI-RADS 1-3) & 11 & 37 & 30 \\
Total & 86 & Lower bound (95\%) & Upper bound (95\%) \\
Statistic & Value & $69 \%$ & $84 \%$ \\
Correct classification & $76 \%$ & $16 \%$ & $31 \%$ \\
Misclassification & $24 \%$ & $78 \%$ & $93 \%$ \\
Sensitivity & $87 \%$ & $36 \%$ & $67 \%$ \\
Specificity & $51 \%$ & $33 \%$ & $64 \%$ \\
False positive rate & $49 \%$ & $6 \%$ & $20 \%$ \\
False negative rate & $13 \%$ & $62 \%$ & $78 \%$ \\
Prevalence & $70 \%$ & $73 \%$ & $89 \%$ \\
Positive predictive value (PPV) & $81 \%$ & $46 \%$ & $81 \%$ \\
Negative predictive value (NPV) & $63 \%$ & 1.27 & 2.52 \\
Positive likelihood ratio (LR+) & 1.79 & 0.13 & 0.47 \\
Negative likelihood ratio (LR-) & 0.25 & 1.38 & 3.51 \\
Relative risk & 2.20 & 2.96 & 17.50 \\
Odds ratio & 7.20 & &
\end{tabular}

Data in cross-tables are counts

Table 5 Diagnostic accuracy of SMG in tested patients with ACR grade A/B versus the result of biopsy or follow-up as the gold standard for lesion classification

\begin{tabular}{llll}
\hline Lesion classification by SMG & Final diagnosis & Benign & Total \\
\cline { 2 - 4 } & Malignant & 7 & 40 \\
\hline Probably malignant (BIRADS 4-6) & 33 & 6 & 9 \\
Probably benign (BIRADS 1-3) & 3 & 13 & 49 \\
Total & 36 & Lower bound (95\%) & Upper bound (95\%) \\
Statistic & Value & $68 \%$ & $91 \%$ \\
Correct classification & $80 \%$ & $9 \%$ & $32 \%$ \\
Misclassification & $20 \%$ & $77 \%$ & $98 \%$ \\
Sensitivity & $92 \%$ & $23 \%$ & $71 \%$ \\
Specificity & $46 \%$ & $30 \%$ & $78 \%$ \\
False positive rate & $54 \%$ & $0 \%$ & $17 \%$ \\
False negative rate & $8 \%$ & $61 \%$ & $86 \%$ \\
Prevalence & $73 \%$ & $71 \%$ & $94 \%$ \\
Positive predictive value (PPV) & $83 \%$ & $36 \%$ & $97 \%$ \\
Negative predictive value (NPV) & $67 \%$ & 1.02 & 2.84 \\
Positive likelihood ratio (LR+) & 1.70 & 0.05 & 0.62 \\
Negative likelihood ratio (LR-) & 0.18 & 1.06 & 5.76 \\
Relative risk & 2.48 & 2.06 & 43.18 \\
Odds ratio & 9.43 & & \\
\hline
\end{tabular}

Data in cross-tables are counts 
Table 6 Diagnostic accuracy of SMG in tested patients with ACR grade C/D versus the result of biopsy or follow-up as the gold standard for lesion classification

\begin{tabular}{llll}
\hline Lesion classification by SMG & Final diagnosis & Benign & Total \\
\cline { 2 - 4 } & Malignant & 11 & 53 \\
\hline Probably malignant (BIRADS 4-6) & 42 & 13 & 21 \\
Probably benign (BIRADS 1-3) & 8 & 24 & 74 \\
Total & 50 & Lower bound (95\%) & Upper bound (95\%) \\
Statistic & Value & $64 \%$ & $84 \%$ \\
Correct classification & $74 \%$ & $16 \%$ & $36 \%$ \\
Misclassification & $26 \%$ & $71 \%$ & $92 \%$ \\
Sensitivity & $84 \%$ & $35 \%$ & $72 \%$ \\
Specificity & $54 \%$ & $27 \%$ & $64 \%$ \\
False positive rate & $46 \%$ & $6 \%$ & $26 \%$ \\
False negative rate & $16 \%$ & $57 \%$ & $78 \%$ \\
Prevalence & $68 \%$ & $68 \%$ & $90 \%$ \\
Positive predictive value (PPV) & $79 \%$ & $41 \%$ & $83 \%$ \\
Negative predictive value (NPV) & $62 \%$ & 1.17 & 2.88 \\
Positive likelihood ratio (LR+) & 1.83 & 0.14 & 1.21 \\
Negative likelihood ratio (LR-) & 0.30 & 2.11 & 0.62 \\
Relative risk & 6.20 & 3.57 \\
Odds ratio & & 18.22 & \\
\hline
\end{tabular}

Data in cross-tables are counts

Table 7 Diagnostic accuracy of CESM in the whole study cohort versus the result of biopsy or follow-up as the gold standard for lesion classification

\begin{tabular}{llll}
\hline Lesion classification by SMG & Final diagnosis & Benign & Total \\
\cline { 2 - 4 } & Malignant & 8 & 92 \\
\hline Probably malignant (BIRADS 4-6) & 84 & 29 & 31 \\
Probably benign (BIRADS 1-3) & 2 & 37 & 123 \\
Total & 86 & Lower bound (95\%) & Upper bound (95\%) \\
Statistic & Value & $87 \%$ & $97 \%$ \\
Correct classification & $92 \%$ & $3 \%$ & $13 \%$ \\
Misclassification & $8 \%$ & $91 \%$ & $100 \%$ \\
Sensitivity & $98 \%$ & $62 \%$ & $89 \%$ \\
Specificity & $78 \%$ & $9 \%$ & $34 \%$ \\
False positive rate & $22 \%$ & $0 \%$ & $5 \%$ \\
False negative rate & $2 \%$ & $62 \%$ & $78 \%$ \\
Prevalence & $70 \%$ & $86 \%$ & $97 \%$ \\
Positive predictive value (PPV) & $91 \%$ & $85 \%$ & $100 \%$ \\
Negative predictive value (NPV) & $94 \%$ & 2.44 & 8.35 \\
Positive likelihood ratio (LR+) & 4.52 & 0.01 & 0.12 \\
Negative likelihood ratio (LR-) & 0.03 & 4.30 & 46.57 \\
Relative risk & 14.15 & 34.97 & 662.91 \\
Odds ratio & 152.25 & &
\end{tabular}

Data in cross-tables are counts 
intense enhancement which corresponded to poor therapy or chemo-resistance in final pathology (true positive), and seven cases showed faint enhancement (false negative) resulting in $31.8 \%$ sensitivity for detection of chemo-resistant tumors post NAC.

The sensitivity, specificity, NPV, and PPV of CESM for prediction of various tumor responses to therapy are $41 \%, 93 \%, 61.8 \%$, and $82 \%$ respectively.

The calculated sensitivity of mammography with US was $82.9 \%$, specificity was $76.5 \%$, and the total accuracy was $81.0 \%$. The PPV and NPV were $89.5 \%$ and $65.0 \%$ respectively.

The calculated sensitivity of CESM was $92.7 \%$, specificity was $82.4 \%$, and the total accuracy was $89.7 \%$. The PPV and NPV were $92.7 \%$ and $82.4 \%$ respectively.

The gold standard references in our study were the histopathology results and follow-up of the patients during the adjuvant therapy.

\section{Discussion}

Contrast-enhanced spectral mammography is an advanced technique of digital mammography done with intravenous injection of an iodinated contrast agent. Dual-energy subtraction imaging exploits the energy dependence of X-ray attenuation through different breast compositions. Post-contrast dual low- and high-energy images are obtained and then the two images are combined and subtracted in order to highlight areas of contrast enhancement. This novel breast imaging technique can be easily implemented clinically using the current digital mammography system with minor modification as well as usage of commercially available iodinated contrast agents.

CESM has been shown to improve the probability of malignancy and BI-RADS assessment in comparison to the conventional mammography alone. The potential clinical applications are localization of disease extent of mammographically equivocal lesions, proper depiction of occult lesions on standard mammography, especially in dense breast, and chemotherapy response monitoring.

CESM implementation is easily accessible in the mammography suite. No specific further training is needed for the radiographers for patient positioning nor for image acquisition, as they are similar to the well-known conventional mammography. CESM examination is well accepted by patients in case of needful complete assessment in contrast to MRI that seems to be unacceptable to some patients. CESM can be performed immediately following mammography, in the same mammography suite with no need for a new appointment and time waste. Moreover, it provides faster imaging when compared to MRI.

Suspicious contrast uptake detected on subtracted CESM images can be easily analyzed retrospectively in correlation to conventional mammograms. The "subtracted" CESM images can be interpreted very easily and rapidly by radiologists and understood by oncologists and surgeons.

The main disadvantage of CESM is the need for intravenous administration of an iodinated contrast agent. CESM is therefore contra-indicated in patients with renal impairment or known allergy or limitations to iodinated contrast agents.

Another disadvantage of CESM as compared with MRI is that CESM is radiation-dependant technique. However, a controlled dose is delivered to the patient, which is approximately equivalent to two conventional mammography examination.

As in all mammography reports, certain essential points should be included in the report for a CESM, including the indication for the examination, comparison if available, findings, ACR BI-RADS lexicon evaluation, and management recommendations. Unique to CESM as compared to conventional mammography reports, background enhancement and lesion contrast enhancement characteristics should be included in the report [12].

Various technical and clinical experience has been acquired with promising results have been published during the last few years regarding CESM.

The temporal subtraction procedure was first acquired with an approach similar to that of breast MRI (Jong et al. [13]; Dromain et al. [14];).

These initial studies, with a limited patient sample, showed that CESM was able to depict tumor angiogenesis in breast cancer.

Jong et al. (2003) examined 22 women with 12 benign and 10 malignant lesions with temporal CESM. Enhancement was noted in $89 \%$ of the invasive cancers. Two false negatives were observed, one case was corresponding to ductal carcinoma in situ and the other one was invasive ductal carcinoma. Among the 12 patients with a benign breast lesion, initially considered worrisome at conventional mammography or US, 5 had nodular enhancement corresponding to 3 fibroadenomas and 2 cases of fibrocystic change with focal intraductal hyperplasia; 7 had no significant enhancement.

Another study was performed by Dromain et al. [14] in which CESM examinations were performed in 20 patients with 22 malignant tumors. The sensitivity of CESM in breast carcinoma detection was $80 \%$. There was a concordance between the histopathology size and the size of enhanced lesion measured on CESM subtracted images. As in the study by Jong et al., most of the cancers showed progressive enhancement without a washout pattern. A poor correlation between the intratumor mean vascular density evaluated on CD34-immunostained histological sections and quantitative characteristics of enhancement kinetics curves was observed, probably due to 
Table 8 Diagnostic accuracy of CESM tested in patients with ACR grade A/B versus the result of biopsy or follow-up as the gold standard for lesion classification

\begin{tabular}{llll}
\hline Lesion classification by SMG & Final diagnosis & Benign & Total \\
\cline { 2 - 4 } & Malignant & 3 & 38 \\
\hline Probably malignant (BIRADS 4-6) & 35 & 10 & 11 \\
Probably benign (BIRADS 1-3) & 1 & 13 & 49 \\
Total & 36 & Lower bound (95\%) & Upper bound (95\%) \\
Statistic & Value & $84 \%$ & $100 \%$ \\
Correct classification & $92 \%$ & $0 \%$ & $16 \%$ \\
Misclassification & $8 \%$ & $84 \%$ & $100 \%$ \\
Sensitivity & $97 \%$ & $49 \%$ & $92 \%$ \\
Specificity & $77 \%$ & $3 \%$ & $43 \%$ \\
False positive rate & $23 \%$ & $0 \%$ & $8 \%$ \\
False negative rate & $3 \%$ & $61 \%$ & $86 \%$ \\
Prevalence & $73 \%$ & $84 \%$ & $100 \%$ \\
Positive predictive value (PPV) & $92 \%$ & $74 \%$ & $100 \%$ \\
Negative predictive value (NPV) & $91 \%$ & 1.56 & 11.38 \\
Positive likelihood ratio (LR+) & 4.21 & 0.01 & 0.26 \\
Negative likelihood ratio (LR-) & 0.04 & 2.27 & 45.19 \\
Relative risk & 10.13 & 15.28 & 890.87 \\
Odds ratio & 116.67 & &
\end{tabular}

Data in cross-tables are counts

Table 9 Diagnostic accuracy of CESM tested in patients with ACR grade C/D versus the result of biopsy or follow-up as the gold standard for lesion classification

\begin{tabular}{llll}
\hline Lesion classification by SMG & Final diagnosis & Benign & Total \\
\cline { 2 - 4 } & Malignant & 5 & 54 \\
\hline Probably malignant (BIRADS 4-6) & 49 & 19 & 20 \\
Probably benign (BIRADS 1-3) & 1 & 24 & 74 \\
Total & 50 & Lower bound (95\%) & Upper bound (95\%) \\
Statistic & Value & $86 \%$ & $98 \%$ \\
Correct classification & $92 \%$ & $2 \%$ & $14 \%$ \\
Misclassification & $8 \%$ & $88 \%$ & $100 \%$ \\
Sensitivity & $98 \%$ & $59 \%$ & $91 \%$ \\
Specificity & $79 \%$ & $6 \%$ & $36 \%$ \\
False positive rate & $21 \%$ & $0 \%$ & $6 \%$ \\
False negative rate & $2 \%$ & $57 \%$ & $78 \%$ \\
Prevalence & $68 \%$ & $83 \%$ & $98 \%$ \\
Positive predictive value (PPV) & $91 \%$ & $85 \%$ & $100 \%$ \\
Negative predictive value (NPV) & $95 \%$ & 2.15 & 10.27 \\
Positive likelihood ratio (LR+) & 4.70 & 0.00 & 0.18 \\
Negative likelihood ratio (LR-) & 0.03 & 3.88 & 84.90 \\
Relative risk & 18.15 & 28.42 & 1220.14 \\
Odds ratio & 186.20 & & \\
\hline
\end{tabular}

Data in cross-tables are counts 


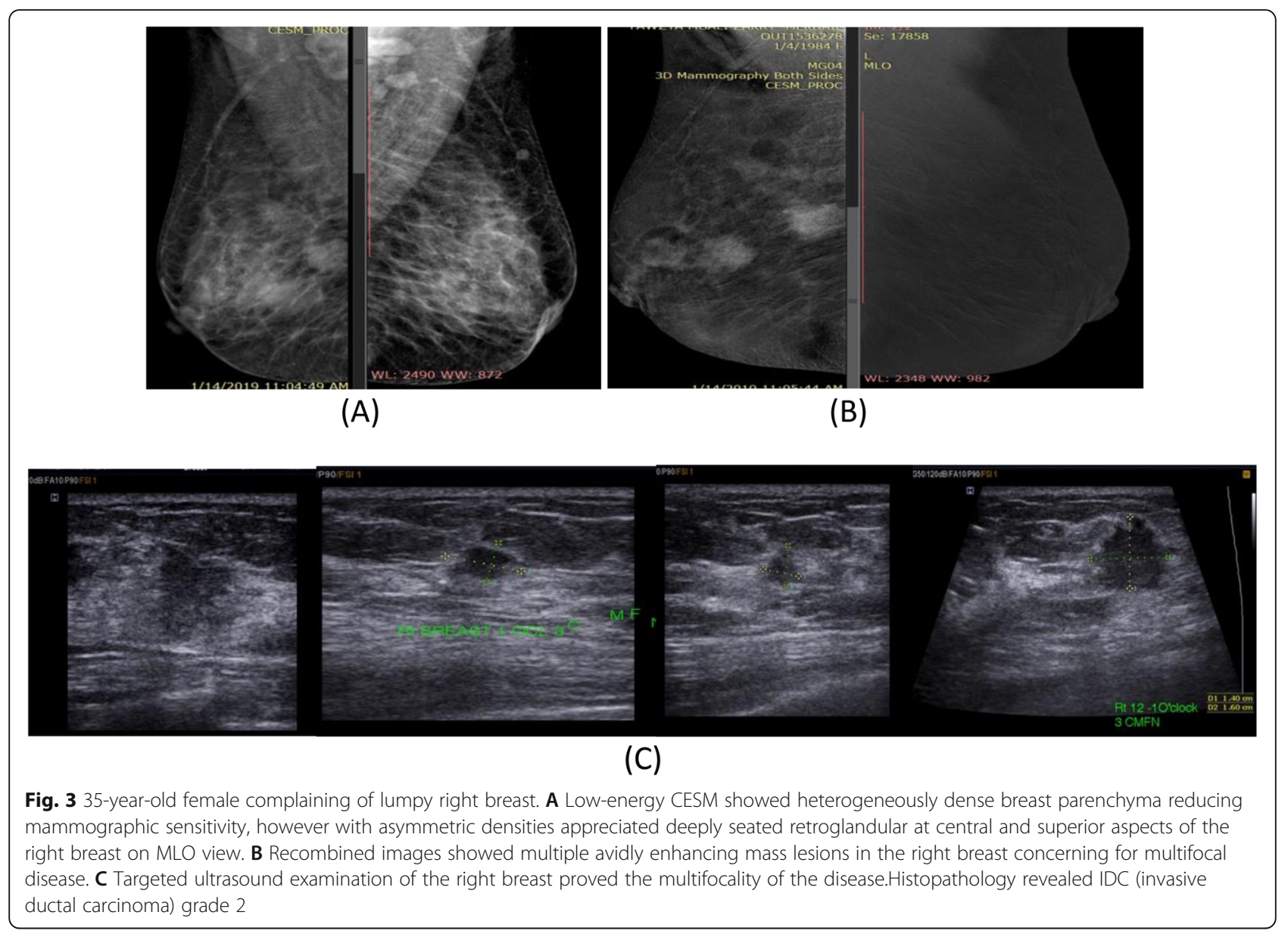

Table 10 Agreement between SMG and CESM as regards the BIRADS classification in the whole study population

\begin{tabular}{|c|c|c|c|c|c|c|c|}
\hline \multirow{2}{*}{$\begin{array}{l}\text { BIRADS by } \\
\text { CESM }\end{array}$} & \multicolumn{6}{|c|}{ BIRADS by SMG } & \multirow[t]{2}{*}{ Total } \\
\hline & BIRADS I & BIRADS II & BIRADS III & BIRADS IV & BIRADS V & BIRADS VI & \\
\hline BIRADS 1 & 0 & 0 & 0 & 0 & 0 & 0 & $0(0.0 \%)$ \\
\hline BIRADS 2 & 3 & 0 & 8 & 17 & 1 & 0 & $29(23.6 \%)$ \\
\hline BIRADS 3 & 0 & 0 & 1 & 0 & 0 & 1 & $2(1.6 \%)$ \\
\hline BIRADS 4 & 3 & 0 & 14 & 19 & 0 & 1 & $37(30.1 \%)$ \\
\hline BIRADS 5 & 0 & 0 & 0 & 4 & 3 & 1 & $8(6.5 \%)$ \\
\hline BIRADS 6 & 1 & 0 & 0 & 0 & 0 & 46 & $47(38.2 \%)$ \\
\hline Total & $7(5.7 \%)$ & $0(0.0 \%)$ & $23(18.7 \%)$ & 40 (32.5\%) & $4(3.3 \%)$ & 49 (39.8\%) & 123 \\
\hline \multicolumn{8}{|c|}{ Inter-method agreement } \\
\hline Weighted kappa & $0.58^{a}$ & & & & & & \\
\hline Standard error & 0.04 & & & & & & \\
\hline $95 \% \mathrm{Cl}$ & 0.49 to 0.67 & & & & & & \\
\hline
\end{tabular}


Table 11 Agreement between SMG and CESM as regards the BIRADS classification in patients with ACR grade A/B

\begin{tabular}{|c|c|c|c|c|c|c|c|}
\hline \multirow{2}{*}{$\begin{array}{l}\text { BIRADS by } \\
\text { CESM }\end{array}$} & \multicolumn{6}{|c|}{ BIRADS by SMG } & \multirow[t]{2}{*}{ Total } \\
\hline & BIRADS I & BIRADS II & BIRADS III & BIRADS IV & BIRADS V & BIRADS VI & \\
\hline BIRADS 1 & 0 & 0 & 0 & 0 & 0 & 0 & $0(0.0 \%)$ \\
\hline BIRADS 2 & 0 & 0 & 3 & 7 & 1 & 0 & $11(22.4 \%)$ \\
\hline BIRADS 3 & 0 & 0 & 0 & 0 & 0 & 0 & $0(0.0 \%)$ \\
\hline BIRADS 4 & 1 & 0 & 4 & 6 & 0 & 0 & $11(22.4 \%)$ \\
\hline BIRADS 5 & 0 & 0 & 0 & 0 & 1 & 0 & $1(2.0 \%)$ \\
\hline BIRADS 6 & 1 & 0 & 0 & 0 & 0 & 25 & $26(53.1 \%)$ \\
\hline Total & $2(4.1 \%)$ & $0(0.0 \%)$ & $7(14.3 \%)$ & $13(26.5 \%)$ & $2(4.1 \%)$ & $25(51.0 \%)$ & 49 \\
\hline \multicolumn{8}{|c|}{ Inter-method agreement } \\
\hline Weighted kappa & $0.59^{\mathrm{a}}$ & & & & & & \\
\hline Standard error & 0.07 & & & & & & \\
\hline $95 \% \mathrm{Cl}$ & 0.45 to 0.73 & & & & & & \\
\hline
\end{tabular}

Data in cross-tables are counts

${ }^{a}$ Moderate agreement

difficulty in performing a quantitative assessment on projection images acquired from breasts under compression.

Indeed, CESM images are projections of the entire breast and enhancement depends on the tumor size. Moreover, enhancement is not exclusively due to the number of vessels but is also most likely related to some other functional parameters such as vessel permeability, especially when using a contrast agent capable of migration to the extracellular fluid space.

In our study, the sensitivity of CESM for the detection of breast carcinoma was $92.7 \%$. There was an excellent concordance between the histopathology size and the size of the enhancement. Most of the cancers showed progressive enhancement without washout.

A multicentric retrospective study was performed by Diekmann et al. [15] aimed at comparing the diagnostic accuracy of CESM with the diagnostic accuracy of mammography alone was performed.

Data were collected at four centers in Europe and North America in 75 women with 85 lesions (17 benign and 68 malignant).

The average sensitivity and specificity across the readers for the probability of cancer increased with mammography plus CESM from 0.81 to 0.86 and 0.62 to 0.66 , respectively. The area under the ROC curve for

Table 12 Agreement between SMG and CESM as regards the BIRADS classification in patients with ACR grade C/D

\begin{tabular}{|c|c|c|c|c|c|c|c|}
\hline \multirow[t]{2}{*}{$\overline{C E S M \_B I R A D S}$} & \multicolumn{6}{|c|}{ SMG_BIRADS } & \\
\hline & BIRADS I & BIRADS II & BIRADS III & BIRADS IV & BIRADS V & BIRADS VI & \\
\hline BIRADS 1 & 0 & 0 & 0 & 0 & 0 & 0 & $0(0.0 \%)$ \\
\hline BIRADS 2 & 3 & 0 & 5 & 10 & 0 & 0 & $18(24.3 \%)$ \\
\hline BIRADS 3 & 0 & 0 & 1 & 0 & 0 & 1 & $2(2.7 \%)$ \\
\hline BIRADS 4 & 2 & 0 & 10 & 13 & 0 & 1 & $26(35.1 \%)$ \\
\hline BIRADS 5 & 0 & 0 & 0 & 4 & 2 & 1 & $7(9.5 \%)$ \\
\hline BIRADS 6 & 0 & 0 & 0 & 0 & 0 & 21 & $21(28.4 \%)$ \\
\hline Total & $5(6.8 \%)$ & $0(0.0 \%)$ & $16(21.6 \%)$ & $27(36.5 \%)$ & $2(2.7 \%)$ & $24(32.4 \%)$ & 74 \\
\hline \multicolumn{8}{|c|}{ Inter-method agreement } \\
\hline Weighted kappa & $0.55^{\mathrm{a}}$ & & & & & & \\
\hline Standard error & 0.06 & & & & & & \\
\hline $95 \% \mathrm{Cl}$ & 0.44 to 0.67 & & & & & & \\
\hline
\end{tabular}

Data in cross-tables are counts

${ }^{\text {a } M o d e r a t e}$ agreement 
Table 13 Agreement between SMG and CESM regarding lesion classification as probably malignant or probably benign in the whole study population

\begin{tabular}{llll}
\hline CESM & SMG & & Total \\
\cline { 2 - 3 } & Probably benign (BIRADS I-III) & Probably malignant (BIRADS IV-VI) \\
\hline Probably benign (BIRADS 1-3) & 12 & 19 & 31 \\
Probably malignant (BIRADS 4-6) & 18 & 74 \\
Total & 30 & 93 & \\
Agreement statistics & & \\
Cohen's kappa ( $K$ ) & & \\
Scott's bias-adjusted kappa (BAK, $\pi$ ) & & $0.19^{\text {a }}$ \\
Bennet's prevalence- and bias-adjusted kappa (PABAK) & $0.19^{\text {a }}$ \\
\hline
\end{tabular}

Data in cross-tables are counts

aslight agreement

${ }^{\mathrm{b}}$ Fair agreement

mammography + CESM using the probability of cancer scale, was higher than the area under the ROC curve mammography alone for all readers.

The study by Lewin et al. [16] is the only published preliminary clinical experience using the dual-energy technique. They studied 26 women with 13 invasive carcinomas. Eight $(85 \%)$ of the invasive carcinoma displayed intense enhancement, 3 showed moderate enhancement, and 1 showed mild enhancement. 5 among those lesions were not detected on conventional mammograms.

Dromain et al., in 2008, have evaluated the dualenergy CESM technique in 120 patients referred for screening mammograms recall with indeterminate mammographic findings.

In our study, we have evaluated 123 patients, For each CESM examination, a pair of low- and high-energy images was acquired using a modified full-field digital mammography system (Senographe DS GE healthcare) with an MLO view at $2 \mathrm{~min}$ and a CC view at $4 \mathrm{~min}$ after injection of $1.5 \mathrm{~mL} / \mathrm{kg}$ of an iodinated contrast agent. Compared to mammography alone, CESM yielded significantly higher sensitivity (93\% vs. $78 \%$ ) and a negative predictive value $(87 \%$ vs.67\%) and had a clearly greater area under the ROC curve. When we compared CESM with the standard examinations (mammography + US), sensitivity was less evident but still exists; however, dual energy had a significant higher specificity than mammography plus US.

CESM is of interest also in the diagnostic setting. It cannot replace mammography but can be helpful in selected cases where clinical and conventional mammography evaluation are still non-conclusive.

In our study, it was of added value in questionable cases with dense breast parenchyma (ACR-C, ACR-D). It was also valuable in the detection of multicentricity and multifocality of breast cancer.

CESM has the potential to increase the cancer detection rate and to improve patient selection for biopsy to avoid unnecessary biopsies.

Basically, the potential indications are closely similar to those of breast MRI. The potential indications for CESM include the evaluation of newly diagnosed breast

Table 14 Agreement between SMG and CESM regarding lesion classification as probably malignant or probably benign in patients with ACR A/B

\begin{tabular}{llll}
\hline CESM & SMG & & Total \\
\cline { 2 - 4 } & Probably benign (BIRADS I-III) & Probably malignant (BIRADS IV-VI) \\
\hline Probably benign (BIRADS 1-3) & 3 & 8 & 11 \\
Probably malignant (BIRADS 4-6) & 6 & 32 & 38 \\
Total & 9 & 40 & \\
Agreement statistics & & & \\
Cohen's kappa (K) & & & $0.12^{\text {a }}$ \\
Scott's bias-adjusted kappa (BAK, $\pi$ ) & & \\
Bennet's prevalence- and bias-adjusted kappa (PABAK) & $0.12^{\text {a }}$ \\
\hline
\end{tabular}

Data in cross-tables are counts

aslight agreement

${ }^{\mathrm{b}}$ Moderate agreement 
Table 15 Agreement between SMG and CESM regarding lesion classification as probably malignant or probably benign in patients with ACR C/D

\begin{tabular}{llll}
\hline & SMG & & Probably malignant (BIRADS 4-6) \\
\cline { 2 - 4 } CESM & Probably benign (BIRADS 1-3) & 11 & Total \\
\hline Probably benign (BIRADS 1-3) & 9 & 42 & 20 \\
Probably malignant (BIRADS 4-6) & 12 & 53 & 54 \\
Total & 21 & & 74 \\
Agreement statistics & & & \\
Cohen's kappa ( $K$ ) & & & \\
Scott's bias-adjusted kappa (BAK, $\pi$ ) & & \\
Bennet's prevalence- and bias-adjusted kappa (PABAK) & $0.22^{{ }^{a}}$ \\
\hline
\end{tabular}

Data in cross-tables are counts

${ }^{\mathrm{a}}$ Fair agreement

cancer, to assess the extent of disease and to evaluate possible contralateral disease, multicentricity, and multifocality, and evaluation of residual disease after a lumpectomy with positive margins, follow-up after chemotherapy, or neoadjuvant therapy to assess for various tumor responses.

Few indications for breast MRI appear superior to CESM: screening of high-risk women (BRCA1/2) due to their sensitivity to radiation exposure, the assessment of response to neoadjuvant chemotherapy as several follow-up imaging examinations are usually needed and will best be performed using a non-radiating imaging method, superior in assessment of axillary lymph nodes and skin assessment.

Our CESM machine is considered among Food and Drug Administration-approved machines.

\section{Interpretation pitfalls and limitations}

It is essential for CESM readers to avoid the pitfall of false negative lesions that have morphologically suspicious findings on low-energy images with no significant enhancement on recombined images. The reader must give adequate consideration to the suspicious morphologic

Table 16 Diagnostic accuracy of marked enhancement on CESM in the whole study cohort

\begin{tabular}{llll}
\hline Lesion classification by SMG & Final diagnosis & Benign & Total \\
\cline { 2 - 4 } & Malignant & 3 & 62 \\
\hline Marked enhancement & 59 & 34 & 61 \\
No to moderate enhancement & 27 & 37 & 123 \\
Total & 86 & Lower bound (95\%) & Upper bound (95\%) \\
Statistic & Value & $68 \%$ & $83 \%$ \\
Correct classification & $76 \%$ & $17 \%$ & $32 \%$ \\
Misclassification & $24 \%$ & $58 \%$ & $77 \%$ \\
Sensitivity & $69 \%$ & $78 \%$ & $98 \%$ \\
Specificity & $92 \%$ & $0 \%$ & $16 \%$ \\
False positive rate & $8 \%$ & $22 \%$ & $41 \%$ \\
False negative rate & $31 \%$ & $62 \%$ & $78 \%$ \\
Prevalence & $70 \%$ & $90 \%$ & $100 \%$ \\
Positive predictive value (PPV) & $95 \%$ & $43 \%$ & $68 \%$ \\
Negative predictive value (NPV) & $56 \%$ & 2.83 & 25.27 \\
Positive likelihood ratio (LR+) & 8.46 & 0.25 & 0.47 \\
Negative likelihood ratio (LR-) & 0.34 & 1.62 & 2.85 \\
Relative risk & 2.15 & 7.54 & 81.31 \\
Odds ratio & 24.77 & &
\end{tabular}

Data in cross-tables are counts 
Table 17 Diagnostic accuracy of marked enhancement on CESM in patients with ACR grade A/B

\begin{tabular}{llll}
\hline Lesion classification by SMG & Final diagnosis & Benign & Total \\
\cline { 2 - 4 } & Malignant & 1 & 22 \\
\hline Marked enhancement & 21 & 12 & 27 \\
No to moderate enhancement & 15 & 13 & 49 \\
Total & 36 & Lower bound (95\%) & Upper bound (95\%) \\
Statistic & Value & $54 \%$ & $80 \%$ \\
Correct classification & $67 \%$ & $20 \%$ & $46 \%$ \\
Misclassification & $33 \%$ & $42 \%$ & $73 \%$ \\
Sensitivity & $58 \%$ & $64 \%$ & $100 \%$ \\
Specificity & $92 \%$ & $0 \%$ & $20 \%$ \\
False positive rate & $8 \%$ & $26 \%$ & $57 \%$ \\
False negative rate & $42 \%$ & $61 \%$ & $86 \%$ \\
Prevalence & $73 \%$ & $87 \%$ & $100 \%$ \\
Positive predictive value (PPV) & $95 \%$ & $26 \%$ & $63 \%$ \\
Negative predictive value (NPV) & $44 \%$ & 1.13 & 50.86 \\
Positive likelihood ratio (LR+) & 7.58 & 0.30 & 0.69 \\
Negative likelihood ratio (LR-) & 0.45 & 1.22 & 2.74 \\
Relative risk & 1.72 & 16.80 & 2.42 \\
Odds ratio & & 103.19 & \\
\hline
\end{tabular}

Data in cross-tables are counts

Table 18 Diagnostic accuracy of marked enhancement on CESM in patients with ACR grade C/D

\begin{tabular}{llll}
\hline Lesion classification by SMG & Final diagnosis & Benign & Total \\
\cline { 2 - 4 } & Malignant & 2 & 40 \\
\hline Marked enhancement & 38 & 22 & 34 \\
No to moderate enhancement & 12 & 24 & 74 \\
Total & 50 & Lower bound (95\%) & Upper bound (95\%) \\
Statistic & Value & $72 \%$ & $90 \%$ \\
Correct classification & $81 \%$ & $10 \%$ & $28 \%$ \\
Misclassification & $19 \%$ & $62 \%$ & $86 \%$ \\
Sensitivity & $76 \%$ & $73 \%$ & $99 \%$ \\
Specificity & $92 \%$ & $0 \%$ & $19 \%$ \\
False positive rate & $8 \%$ & $13 \%$ & $35 \%$ \\
False negative rate & $24 \%$ & $57 \%$ & $78 \%$ \\
Prevalence & $68 \%$ & $88 \%$ & $100 \%$ \\
Positive predictive value (PPV) & $95 \%$ & $49 \%$ & $81 \%$ \\
Negative predictive value (NPV) & $65 \%$ & 2.40 & 34.69 \\
Positive likelihood ratio (LR+) & 9.12 & 0.16 & 0.44 \\
Negative likelihood ratio (LR-) & 0.26 & 1.72 & 4.21 \\
Relative risk & 2.69 & 8.14 & 149.11 \\
Odds ratio & 34.83 & &
\end{tabular}




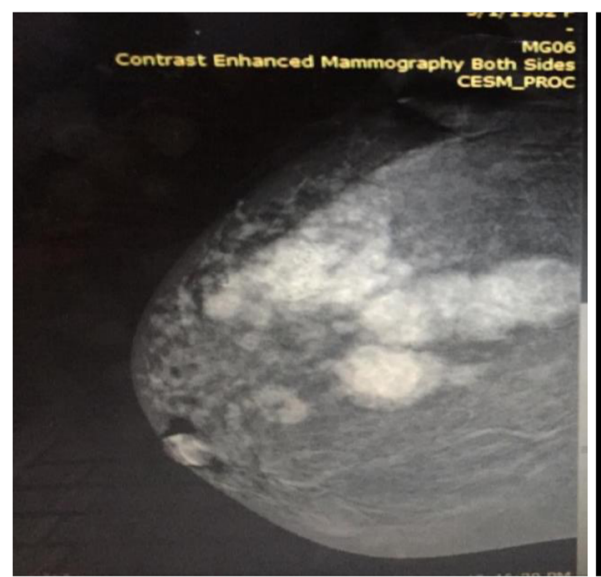

(A)

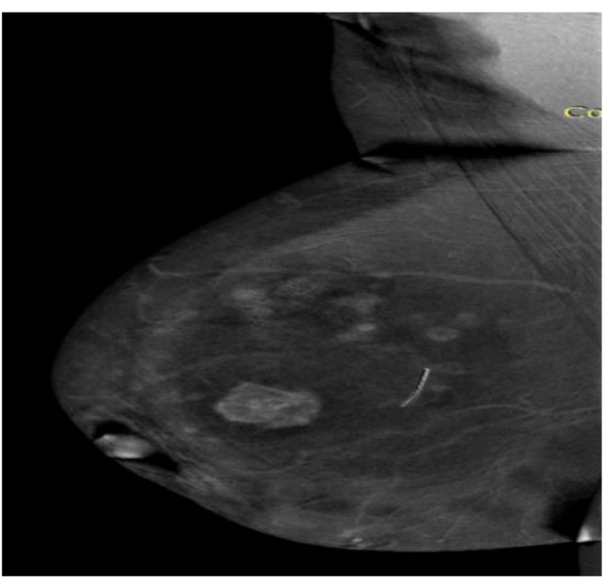

(B)

Fig. 4 37-year-old, pathologically proven multicentric disease: IDC. A MLO recombined image prior to NAC revealed locally advanced disease with extensive mass and non-mass enhancement occupying most of the superior and central right breast. B Post 6 cycles of NAC showing marked disease regression and partial response

findings on the low-energy images when ultimately determining the appropriate BI-RADS category [12].

\section{Our study has some limitations and pitfalls}

Few benign-looking lesions like fibroadenoma were considered false positive results as they displayed contrast enhancement. A study by Badr et al. [17] showed enhancement in $33 \%$ of 27 benign lesions. Jochelson et al. [18] observed 2 false positive findings in 52 cases. Lobbes et al. [19] detected 5 false positive findings in a study including 113 women recalled from screening, most of them. Most of these false positive lesions are caused by fibroadenomas

Faint ring enhancement occurred with one lesion is mucinous carcinoma, and considered a false negative result.

Athough two experienced radiologists evaluated the digital mammography and CESM findings, intraobserver and interobserver variability were not evaluated.

\section{Conclusion}

CESM is a potential promising technique in the evaluation of patients with mammographically inconclusive findings especially those with mammographically dense breasts.

CESM is feasible and has an important role in proper characterization of breast lesions especially in dense breast parenchyma. CESM showed higher sensitivity and specificity compared to digital mammography with ultrasound.

CESM is cosidered a cost-effective, good, alternative to dynamic contrast-enhanced MRI (DCE-MRI) for the staging of newly diagnosed breast cancer.

\section{Abbreviations}

CESM: contrast-enhanced spectral mammographyBI-RADSBreast imaging reporting and data systemCCCranio-caudalMLOMedio-lateral obliqueUSUltrasoundROCReceiving operating characteristicPRPartial responseCRComplete responseSDStable diseasePDProgressive diseasePPVPositive predictive valueNPVNegative predictive valueNACNeoadjuvant chemotherapyDCE-MRIDynamic contrast-enhanced magnetic resonance imagingIDCInvasive ductal carcinoma

\section{Acknowledgements}

This research was carried out at Ain Shams University hospital which is fully equipped with dedicated machines for proper breast imaging. We want to thank our colleagues who helped us to do such research work.

\section{Authors'contributions}

NC collected patient data from the dedicated cases and was responsible for correspondence to the journal. AA performed the statistical analysis and participated in manuscript drafting. ME participated in writing the manuscript and its revision. The authors have read and approved the manuscript.

\section{Funding}

No funding sources.

\section{Availability of data and materials}

The data used and analyzed during this study is available from the corresponding author on reasonable request.

\section{Declarations}

\section{Ethics approval and consent to participate}

The protocol was reviewed and approved by the local ethical committee of "Research Ethics Committee at the Faculty of Medicine, Ain Shams University." It stated that no formal ethics approval was required in a retrospective study, and so, no reference number was given by the IRB.

\section{Consent for publication}

Although this research is based on a retrospective study, written consent for publication was obtained for these cases.

Competing interests

No financial or non-financial competing interest. 
Received: 31 March 2021 Accepted: 16 July 2021

Published online: 27 July 2021

\section{References}

1. Skaane P, Balleyguier C, Diekmann F, Diekmann S, Piguet JC, Young K, Niklason LT (2005) Breast lesion detection and classifi cation: comparison of screen-film mammography and full-field digital mammography with softcopy reading-observer performance study. Radiology 237(1):37-44. https:// doi.org/10.1148/radiol.2371041605

2. Bick U, Diekmann F (2007) Digital mammography: what do we and what don't we know? Eur Radiol 17(8):1931-1942. https://doi.org/10.1007/s00330007-0586-1

3. Pisano ED, Gatsonis C, Hendrick E, Yaffe M, Baum JK, Acharyya S, Conant EF, Fajardo LL, Bassett L, D'Orsi C, Jong R, Rebner M (2005) Digital Mammographic Imaging Screening Trial (DMIST) Investigators Group. Diagnostic performance of digital mammography versus film mammography for breast-cancer screening. N Engl J Med 353(17):17731783. https://doi.org/10.1056/NEJMoa052911

4. Diekmann F (2005) Digital mammography using iodine-based contrast media initial clinical experience with dynamic contrast medium enhancement. Invest radiol 40(7):397-404. https://doi.org/10.1097/01.rli. $0000167421.83203 .4 \mathrm{e}$

5. Diekmann F, Fredenberg E, Lundqvist $M$ et al (2008) Contrast agents in digital mammography: a new diagnostic option resulting from the use of photon-counting detectors. In: RSNA abstract book, p 121

6. Mori M, Akashi-Tanaka S, Suzuki S, Daniels MI, Watanabe C, Hirose M, et al (2017) Diagnostic accuracy of contrast-enhanced spectral mammography in comparison to conventional full-field digital mammography in a population of women with dense breasts Breast Cancer. Breast Cancer 24(1):104-110

7. Dromain C, Thibault F, Muller S, Rimareix F, Delaloge S, Tardivon A, Balleyguier C (2011) Dual-energy contrast-enhanced digital mammography: initial clinical results. Eur Radiol 21(3):565-574. https://doi.org/10.1007/s0033 0-010-1944-y

8. American College of Radiology (2007) ACR practice guidelines for the performance of magnetic resonance imaging (MRI) of the breast. Arch Oncol 15(1-2):37-34

9. D'Orsi CJ, Sickles EA, Mendelson EB, Morris EA (2013) Acr BI-RADS ${ }^{\oplus}$ Atlas, breast imaging reporting and data system. American College of Radiology, Reston

10. Morris EA, Comstock CE, Lee $\mathrm{CH}$ et al (2013) ACR BI-RADS ${ }^{\oplus}$ magnetic resonance imaging. In: In: ACR BI-RADS ${ }^{\circledR}$ atlas, breast imaging reporting and data system. American College of Radiology, Reston

11. Eisenhauera EA, Therasseb P, Bogaertsc J, Schwartzd LH, Sargente D, Fordf R et al (2009) A new response evaluation criteria in solid tumours: revised RECIST guideline (version 1.1). Eur J Cancer 45(2):228-247. https://doi.org/1 0.1016/j.ejca.2008.10.026

12. Perry H, Phillips J, Dialani V, Slanetz PJ, Fein-Zachary VJ, Karimova EJ, Mehta TS (2019) Contrast-enhanced mammography: a systematic guide to interpretation and reporting. AJR 212(1):222-231. https://doi.org/10.2214/A JR.17.19265

13. Jong RA, Yaffe MJ, Skarpathiotakis M et al (2003) Contrast-enhanced digital mammography: initial clinical experience. Radiology 228:842-850

14. Dromain C, Balleyguier C, Muller S et al (2006) Evaluation of tumor angiogenesis of breast carcinoma using contrast-enhanced digital mammography. AJR Am J Roentgenol 187:W528-W537

15. Diekmann F, Diekmann S, Jeunehomme F, Muller S, Hamm B, Bick U (2005) Digital mammography using iodine-based contrast media. Invest Radiol 40: 397-404

16. Lewin JM, Isaacs PK, Vance V, Larke FJ (2003) Dual-energy contrastenhanced digital subtraction mammography: feasibility. Radiology 229:261268

17. Badr S, Laurent N, Regis C et al (2014) Dual-energy contrast-enhanced digital mammography in routine clinical practice in 2013. Diagn Interv Imaging 95(3):245-258. https://doi.org/10.1016/j.diii.2013.10.002

18. Jochelson MS, Dershaw DD, Sung JS, Heerdt AS, Thornton C, Moskowitz CS, Ferrara J, Morris EA (2013) Bilateral contrast-enhanced dual-energy digital mammography: Feasibility and comparison with conventional digital mammography and MR imaging in women with known breast carcinoma. Radiology 266(3):743-751. https://doi.org/10.1148/radiol.12121084

19. Lobbes MB, Lalji U, Houwers J, Nijssen EC, Nelemans PJ, van Roozendaal L, Smidt ML, Heuts E, Wildberger JE (2014) Contrast-enhanced spectral mammography in patients referred from the breast cancer screening programme. Eur Radiol 24(7):1668-1676. https://doi.org/10.1007/s00330014-3154-5

\section{Publisher's Note}

Springer Nature remains neutral with regard to jurisdictional claims in published maps and institutional affiliations.

\section{Submit your manuscript to a SpringerOpen ${ }^{\circ}$ journal and benefit from:}

- Convenient online submission

- Rigorous peer review

- Open access: articles freely available online

High visibility within the field

- Retaining the copyright to your article

Submit your next manuscript at $\boldsymbol{\nabla}$ springeropen.com 